\title{
Modelo AIM para la enseñanza-aprendizaje con estudiantes de modalidad semipresencial-distancia en la Universidad Nacional de Educación
}

\section{Teaching-learning AIM model for semi presence and distance students at the National University of Education}

\author{
Leonardo Zambrano-Vacacela ${ }^{1 *}$ y Mario Yautibug-Chimbolema ${ }^{1}$ \\ ${ }^{1}$ Universidad Nacional de Educación, Ecuador \\ *leozamv@ hotmail.com
}

DOI: https://doi.org/10.26871/killkana_social.v4i1.629

\begin{abstract}
Resumen
La modalidad semipresencial-distancia en la Educación Superior, demanda la creación de nuevas estrategias que estimulen la enseñanza y el aprendizaje. Este artículo expone una investigación sistematizada sobre la ejecución del enfoque de Aula Invertida articulada con la Motivación; se plantea un marco teórico que sustenta el modelo AIM en el desarrollo y construcción de una práctica pedagógica generada en la Universidad Nacional de Educación (UNAE) Javier Loyola, Azogues, Cañar, Ecuador, con estudiantes que son docentes del sistema educativo fiscal y particular; pertenecientes a quinto ciclo en la cátedra El Medio Natural, carrera de Educación General Básica (EGB) modalidad a distancia. La propuesta responde a la carencia de metodologías relacionadas con el conectivismo que los profesores necesitan para desempeñar la docencia con estudiantes adultos; y se fundamenta en el Modelo pedagógico UNAE; para lo cual se recurre al Modelo AIM como didáctica de enseñanza en la educación híbrida; por lo que se planifica actividades autónomas en plataforma virtual, praxis en las instituciones educativas y jornadas presenciales en la universidad; espacios que forjan el diálogo abierto, trabajo colaborativo y cooperativo en pro de consolidar aprendizajes mediante las técnicas de feedback, feedforward y motivación; pretendiendo alcanzar objetivos planteados en la malla curricular, desarrollar competencias, generar y aplicar en contextos educativos los conocimientos desaprendidos, aprendidos o fortalecidos en la universidad. Apoyados en la investigación cuantitativa y cualitativa se proyecta obtener resultados que muestren la percepción de los estudiantes-docentes sobre la ejecución del modelo AIM en el aula al finalizar el ciclo.
\end{abstract}

Palabras clave: Aula Invertida, Distancia, Educación, Motivación, Semipresencial.

\begin{abstract}
The semi presence-distance modality in Higher Education requires the creation of new strategies to stimulate teaching and learning. This article presents systematized research on the Flipped Classroom approach implementation combined with Motivation. It proposes a theoretical framework that supports the AIM model in the development and creation of a pedagogical practice generated at the National University of Education (UNAE) Javier Loyola, Azogues, Cañar, Ecuador. This study involves students who are teachers public and private education system, attending to the fifth cycle of El Medio Natural course, in the General Basic Education (EGB) distance mode. The proposal responds to the lack of methodologies related to the connectivism teachers need to work with adult students. It is based on the UNAE pedagogical model, which uses the AIM model as a teaching methodology in hybrid education. Autonomous activities on virtual platforms, praxis in the educational institutions and face-to-face conferences at the university are planned, as opportunities that promote open dialogue, collaborative and cooperative work, to consolidate learning through feedback, feedforward and motivation techniques. The objective to be achieved is established in the curriculum, which purpose is to develop skills, and to generate and apply, in educational contexts, the unlearned, learned or strengthened knowledge in the university. Supported by quantitative and qualitative research, results intend to show the student-teachers perception of the AIM model execution in the classroom, at the end of the term.
\end{abstract}

Keywords: Flipped classroom, Distance, Education, Motivation, Blended.

\section{Introducción}

El avance de la tecnología influye en el sistema educativo; los textos o recursos físicos dejan de ser im- 
prescindibles, a tal punto que al crear herramientas, estrategias o metodologías deben tener conexión con la era de la Tecnología de la Información y Comunicación (TIC) (Gaviria-Rodríguez, Arango-Arango, ValenciaArias, y Bran-Piedrahita, 2019).

Esto genera un reto para los educadores; demandando la creación de nuevas alternativas para aplicarlas en el proceso enseñanza-aprendizaje (Reyes, Vera, y Colina, 2014). Consecuentemente, la sociedad de la información provee herramientas interactivas que fortalecen el conocimiento; por lo que es necesario replantear el proceso educativo desde la corriente conectivista que propone una instrucción cimentada en la web 2.0 (Sobrino, 2014).

Sin embargo, los docentes tienen limitaciones al momento de impartir la cátedra en la modalidad semipresencial-distancia; puesto que no existen modelos específicos que produzcan aprendizajes significativos en la educación presencial-virtual; reduciendo la calidad de la educación en estos contextos.

La Organización de las Naciones Unidas para la Educación la Ciencia y la Cultura y el Fondo de la Naciones Unidas para la Infancia, coinciden en el planteamiento de estrategias metodológicas de información, conocimiento, sabiduría y competencias, basadas en recursos didácticos con sustento teórico y práctico; de tal forma que se desarrolle habilidades y destrezas mediante el uso de la TIC como complemento a las necesidades académicas; garantizando calidad educativa; derecho que compete a los seres humanos durante su existencia (UNESO, 2019; UNICEF, 2019).

De estos antecedentes surgen las preguntas: ¿Qué tiempo otorgar a los estudiantes para el cumplimiento de actividades en plataforma virtual? ¿Qué actividades fortalecen las competencias que los estudiantes necesitan? ¿Cómo planificar la jornada presencial? ¿Cómo orientar (presencial-virtual) al estudiante para fortalecer sus aprendizajes? ¿Qué estrategias utilizar para motivar a la población adulta? Resumida en una sola pregunta ¿El modelo AIM apoya, fortalece y motiva el proceso enseñanza-aprendizaje en la educación superior semipresencial-distancia?

Actualmente, es un reto lograr la interacción de los estudiantes en el proceso (Dutra, De Sousa, y Antonio, 2017); motivo por el que los establecimientos educativos, mediante el personal docente buscan alternativas que impulsen la interactividad profesorado-alumnado ambivalentemente en los espacios ofrecidos por la TIC; en donde el discente sea el primer accionista del proceso orientación-aprendizaje y el docente un guía (Álvarez, Peréz, , y and, 2015).

El objetivo de esta investigación es proponer un modelo fundamentado en Aula Invertida y Motivación (AIM), para que el profesorado acceda y oriente los conocimientos que exige el currículo en el que trabaja. Afirmando que el modelo AIM es una alternativa que provee herramientas metodológicas para apoyar al claustro en la educación semipresencial-distancia.
La investigación fue realizada en la Universidad $\mathrm{Na}$ cional de Educación (UNAE); con alumnos de la carrera de Educación General Básica, modalidad a distancia; considerando que esta población cumple con las características imprescindibles para la aplicación del modelo AIM, para lo cual se trabajó en las variables del enfoque de aula invertida y motivación.

\section{Marco teórico}

Integrar las TIC al proceso educativo no es sinónimo de calidad, debe existir una combinación complementada entre lo didáctico, tecnológico y motivacional de forma óptima y creativa; por consiguiente, el profesorado que utiliza medios virtuales para efectuar la docencia, necesita la capacidad de promover el aprendizaje para la construcción del conocimiento (Duarte-Herrera, Montalvo, y Valdes, 2019).

El modelo didáctico AIM, propone la aplicación de una metodología estructurada en Aula Invertida (A.I) o Flipped Classroom paralelamente a la motivación; planteando una educación híbrida (blended learning o blearning); entre lo presencial, virtual (Schmitz y dos Reis, 2018; Paredes y Dias, 2012) y motivacional; a fin de brindar fortalezas que ayuden a superar adversidades, alcanzar metas y expectativas en el ámbito académico semipresencialdistancia (Rojas -Lamorú y Matos-Columbié, 2015).

\subsection{Aula invertida o Flipped Classroom}

Autores como (Zainuddin y Halili, 2016) explican que el Aula Invertida es el enfoque que utiliza herramientas sincrónicas o asincrónicas de manera presencial y a distancia mediante plataformas virtuales, apoyándose en las virtudes proporcionadas por la web 2.0. Mediante su aplicación se provee al estudiantado materiales y recursos anticipadamente para resolver actividades antes de la jornada presencial; proporcionando la responsabilidad de generar hábitos como: autoaprendizaje, autodisciplina y autorregulación, acciones que conllevan a la adquisición de nuevos conocimientos (Gaviria-Rodríguez y cols., 2019).

Además, el enfoque A.I. propicia aprendizajes colaborativos, cooperativos y constructivos; mejora el manejo tecnológico, fomenta la interacción docente-estudiante, estudiante-estudiante (Hernández-Silvaa y Tecpan, 2017); se adapta a las necesidades de aprendizaje y tiempo, motiva la investigación, proporciona variedad de recursos y herramientas, fortalece la reflexión y autocrítica, entre otros (Aguayo, Bravo, Nocetti, Concha, y Aburto, 2019).

Por esta razón, (Tristán y Mendoza, 2016), afirman que las actividades deben ser elaboradas de manera que el discente comprenda la tarea para encontrar las soluciones a los problemas presentados; organice los procedimientos enfocándose en las diversas alternativas o resultados y concrete lo planificado, lo mismo que fundamenta Bloom en su taxonomía.

Adicionalmente, los trabajos deben estar orientados a la solución de problemáticas cotidianas o a la ejecución de proyectos (Gaviria-Rodríguez y cols., 2019), para salir de la enseñanza tradicional que el profesorado utiliza en el aula 
y aprovechar los métodos interactivos de las herramientas multimedia en la sociedad del conocimiento. (MartínezOlvera, Esquivel-Gámez, y Martínez, 2014)

\subsection{Motivación}

Para autores como (Parrales y Solórzano, 2014), la motivación en el ser humano es el impulso para ejecutar acciones pertinentes que conllevan a alcanzar objetivos trazados; es decir, la ausencia del estímulo provoca un bajo desempeño en las actividades encomendadas.

De la misma manera, (Pinchao, 2016) afirma que la motivación es importante en las personas, debido a que incita a direccionar e intensificar esfuerzos para adquirir los conocimientos; por eso es necesario generar ambientes educativos que fomenten la estimulación, tomando en cuenta que existe la intrínseca y extrínseca (Valenzuela, Valenzuela, Silva-Peña, Gómez, y Precht, 2015).

En el ámbito educativo, la motivación intrínseca es el interés que el alumnado demuestra en las tareas, evidenciado en su esfuerzo; induce a demostrar la voluntad por desarrollar las actividades, profundizando los conocimientos; mientras que la extrínseca enfatiza en lo externo (Salgado, Lería, Pilar, Gajardo, y Olivares, 2017), busca recibir reconocimiento visible como: la obtención de una buena calificación o un estímulo verbal (Parrales y Solórzano, 2014).

En ese sentido, las estrategias metodológicas en el campo de la educación deben encaminarse a incentivar a los actores inmersos en el proceso, a fin de satisfacer demandas propuestas por el sistema actual, transformando la formación tradicional. Para esto, existen varias estrategias didácticas motivacionales que pueden utilizarse en el enfoque de Aula Invertida; sin embargo, el profesorado debe tener la experticia (conocimiento-experiencia-sabiduría) para seleccionarlas de acuerdo a las necesidades educativas; por lo que es importante analizar, generar condiciones, ejecutar, mantener y promover la reflexión en el contexto que se desarrolla el aprendizaje.

\subsection{Modelo AIM}

El Modelo Aula Invertida y Motivación (AIM), guía al personal docente para la elaboración de actividades bajo las variables mencionadas. Desde esta perspectiva se plantea 5 etapas sistemáticas.

\subsubsection{Búsqueda de información}

Se propone trabajar a través de actividades autónomas virtualizadas de forma descriptiva, orientativa; de tal manera que el alumnado pueda demostrar los conocimientos previos (Montilla y Arrieta, 2015), las instrucciones deben contener la competencia a obtener (técnica motivacional), recursos bibliográficos, producto a presentar, parámetros con los que va a ser evaluado y tiempo estimado para el desarrollo de la aptitud.

Gracias a la tecnología es posible transmitir, retransmitir, almacenar y recuperar la información; posibilitando el acceso a un sinnúmero de referencias sobre el tema planteado (Reyes y cols., 2014); no obstante, el estudiantado debe tener la destreza y la madurez intelectual para seleccionar el contenido pertinente, siguiendo normas éticas que conllevan a la honestidad académica.

Cabe recalcar que en la web existen muchas referencias sobre temas a investigar; sin embargo, no se puede confiar en toda la información; hay páginas específicas recomendadas para la educación. Para (Kriscautzky y Ferreiro, 2014) los estudiantes no cuentan con las destrezas necesarias para la búsqueda de contenido veraz; por lo que el profesorado debe proporcionar páginas de internet académicas accesibles y fidedignas.

\subsubsection{Generación de conocimientos}

El conocimiento es la capacidad para identificar, reflexionar, interiorizar, ampliar y transformar la información; lo cual ayuda a encontrar posibles soluciones a problemáticas que se presentan en el contexto.

El personal docente debe acceder a distintos recursos didácticos como: vídeos, juegos online, audios, textos literarios, dibujos, casos, entre otros; para plantear preguntas y actividades relacionadas con problemáticas cotidianas; orientando a la recuperación de conocimientos previos o adquiridos que provoquen interés o curiosidad.

Las tareas deben encaminarse a generar o fortalecer competencias; para lo cual se tiene a disposición técnicas de explicación, análisis de textos, comparaciones, deducciones, praxis, sistematización de información; para que el educando aprenda experimentando. Posteriormente, el profesor verificará y monitoreará los conocimientos asimilados e interiorizados; mediante la evaluación de actividades o cuestionarios tipo reactivo en plataforma virtual.

Finalmente, realizará una retroalimentación específica, orientativa y al mismo tiempo constructiva; es decir, ejecutar un feedback para detectar, señalar errores o aciertos que ayuden a mejorar la tarea en ese momento y un feedforward para orientar, motivar y apoyar a seguir investigando sobre el tema en estudio, evitando errores en trabajos ulteriores (Canabal y Margalef, 2017; De la TorreLaso, 2019) .

\subsubsection{Adquisición de competencias}

Para responder a las necesidades sociales es necesario producir aprendizajes significativos mediante la experimentación de los conocimientos alcanzados; de tal manera que sean comprendidos desde la realidad humana y el crecimiento personal del estudiante.

Frente a estos criterios el personal docente construirá actividades que encaminen a utilizar habilidades, competencias y destrezas logradas. Además, planificará tareas de praxis que favorezcan el desarrollo cognitivo en base a una problemática. Pueden ser debates, talleres, mesas redondas, conferencias, ponencias, acrósticos, carteles, material de lectura creativa, entre otros; que le permitan exponer el trabajo desarrollado. 


\subsubsection{Aplicación de conocimientos}

La cognición es producto de un aprendizaje adquirido mediante un proceso sistemático; por lo tanto, la aplicación debe ser una metamorfosis del saber teórico a la praxis; convirtiéndose en un deber moral de la persona ejecutar lo aprendido para beneficio de la sociedad (Espín y Zambrano, 2018).

En esta etapa el discente debe demostrar las competencias alcanzadas de manera inter y transdisciplinar; exponiendo ante la comunidad su imaginación, ingenio, sentimientos y emociones desarrolladas durante las fases analizadas anteriormente. Para esto puede utilizar las técnicas de socialización, reflexión, debate, compromisos, exposiciones, talleres, foros, ponencias, entre otros.

\subsubsection{Ejecución de Estrategias Motivacionales}

Dentro de las estrategias motivacionales se encuentra la aplicación del feedforward y mensajería en plataforma, por esta razón deben ser orientadas de manera constructiva y re-orientativa; adicionalmente como parte indispensable del modelo AIM, es necesario impartir charlas de motivación con argumentos de interés de la población a la que se ofrece la cátedra; estos temas deben ser seleccionados con base a un diagnóstico inicial.

Al mismo tiempo, el modelo AIM recomienda tratar los siguientes temas: Actitud, que según (Beltrán, 1998) influye en la respuesta que tiene el discente ante el aprendizaje; Estrategias para organizar la vida familiar-estudio, con esto se proporciona técnicas para planificar, optimizar tiempo y recursos, que según (Fuentes y Pérez, 2019) la familia es parte de la colectividad que influye en el aspecto académico del estudiante; y la Inteligencia Emocional que según (Goleman, 2007), fortalecer y conocer este aspecto es sustancial para alcanzar el éxito en la sociedad actual.

Cabe destacar que parte de las estrategias motivacionales precisa realizar dinámicas grupales para adultos o ejercicios que fomenten y despierten la atención del alumnado, considerando la edad y carga horaria; entendiendo que laboran durante la semana y provienen de lugares lejanos a la universidad.

\section{Metodología}

\subsection{Enfoque}

La investigación se fundamenta en un método híbrido (cualitativo-cuantitativo); proceso argumentado en una experiencia de aplicación de un modelo con alcance descriptivo basado en resultados.

\subsection{Contextualización y caracterización de la población}

La presente investigación se desarrolló en la etapa marzo-agosto 2019, con estudiantes de quinto ciclo de la carrera Educación General Básica, modalidad a distancia en la Universidad Nacional de Educación, población que ingresa al primer período para profesionalizarse; previamente a la ejecución se informó sobre el proceso que consiste en la aplicación de Aula Invertida como parte del modelo pedagógico UNAE.

Para este estudio colaboraron 123 educandos pertenecientes a la asignatura El medio natural: Problematización y diversidad epistemológica. Los participantes en la investigación son docentes en ejercicio, con mínimo 5 años de experiencia, edad promedia entre los 25-55 años, laboran en instituciones educativas públicas y privadas en las provincias de Cañar, Azuay y El Oro.

Al hacer una descripción cualitativa, se afirma que la mayoría son migrantes digitales; tuvieron que adaptarse a la tecnología. Según los resultados se aprecia que el 70,1\% son de sexo femenino y el $29,9 \%$ masculino. En estado civil, el $29,4 \%$ son solteros, el $62,2 \%$ casados y el $8,4 \%$ divorciados. En el aspecto de acceso a internet, el 74,6\% tienen red permanentemente mediante planes mensuales en dispositivo móvil y hogar; el 24,6\% navegan de manera esporádica, porque no cuentan con datos en los móviles, pero lo hacen en las instituciones educativas y/o casa, y el $0,8 \%$ no cuentan con el servicio, utilizando centros de cómputo particulares o públicos. Respecto al lugar de procedencia, la provincia que más estudiantes posee es Azuay con 74,8 \%; el 21,7 \% pertenecen a Cañar y un 3,5\% a El Oro. El rango de edad con mayor porcentaje es 25-30 años con $29,7 \%$; seguido de $31-35$ con $26,3 \%$; 36-40 con $18,6 \%$; $41-45$ con $15,3 \%, 46-50$ con $6,8 \%$ y finalmente $51-55$ con $3,4 \%$ del total de la población participante.

\subsection{Técnicas de recolección de datos}

Para reunir información se filmó las clases fomentando el diálogo interactivo con charlas de motivación sobre temas específicos como: actitud, familia e inteligencia emocional; a lo que se suma dinámicas grupales. Posteriormente, se observó el vídeo y se analizó la reacción del alumnado a lo largo de toda la jornada presencial. Finalmente se realizó entrevistas a los educandos para obtener una interpretación cualitativa sobre el modelo AIM.

Al finalizar el ciclo se elaboró y solicitó a los estudiantes contestar una encuesta cerrada que permite obtener información y datos estandarizados mediante la observación indirecta (Casas, Repullo, y Donado, 2003); con escala tipo Likert que proporciona diferentes opciones de respuesta (Bozal, 2006).

Para hacer un diagnóstico sobre el cumplimiento de los puntos relevantes de Aula invertida y motivación, la encuesta se divide en seis partes articuladas: tiempo para presentar trabajo autónomo, aprendizajes y competencias adquiridas, trabajo presencial, orientación académica docente, estrategias motivacionales y percepción de la eficacia del Modelo AIM.

\subsection{Procesamiento de análisis}

Para analizar resultados se apoyó en el programa SPSS que cubre necesidades de cálculo estadístico (Universidad de Córdova, 2010). En el software se ingresaron datos con once variables obtenidas del cuestionario aplicado al total 
de la población, consiguiendo resultados cuantitativos. De la misma manera, se explica la ejecución de cada etapa en respuesta a las variables planteadas.

\section{Resultados}

El modelo AIM está planteado para estudiantes que tiene las carreras a distancia de la Universidad Nacional de Educación; población que demanda técnicas de enseñanza para personas adultas que no asistieron a estudios superiores; son padres de familia, poseen un trabajo que cumplen para mantenerse económicamente y su edad oscila entre los 25 y 55 años.

Frente a esta realidad el propósito del modelo AIM es adaptar al enfoque de aula invertida estrategias motivacionales que fomenten el aprendizaje e incentiven la asistencia a la universidad; de tal manera que articulen la malla curricular con aspectos motivacionales-emocionales útiles para el desempeño en todos los ámbitos.

Se trabajó entre dos docentes con habilidades en creación de material didáctico en ambientes virtuales y en estrategias motivacionales para estudiantes. El tiempo de aplicación fue seis semanas de clase para cada curso; con un total de cuatro paralelos.

Cabe recalcar que las actividades para la semana deben ser distribuidas equitativamente; lo óptimo es asignar 2H30' por día durante cuatro consecutivos; dejando el quinto día libre para que el personal docente ejecute la técnica del feedforward; finalmente, el sexto día, el alumno debe asistir a la jornada presencial para consolidar los aprendizajes adquiridos.

\subsection{Planificación del Aprendizaje autónomo y Aprendiza- je experimental}

Con base a la planificación desarrollada y ejecutada en el componente de trabajo autónomo y de praxis el $80,5 \%$ de los estudiantes manifiestan que están de acuerdo con los tiempos establecidos para realizar las actividades en plataforma virtual; mientras que el 8,2\% expresan que no es suficiente, por esta razón se demoran en el desarrollo de las tareas. Sin embargo el 11,4\% del estudiantado se encuentra en un estado neutral; es decir no opinan si el tiempo es suficiente o insuficiente; destacando que el porcentaje de alumnos que no presentan las tareas; según las entrevistas desarrolladas a los mismos estudiantes; tienen otras dificultades como: falta de hábitos de estudio, exceso de carga administrativa en sus lugares de trabajo, problemas familiares y emocionales, entre otros. En definitiva, el modelo AIM cumple con los objetivos planteados en las etapas de búsqueda de información, generación de conocimiento que se producen y fortalecen por medio del hacer y experimentar.

\subsection{Aprendizajes y competencias adquiridas}

Este ámbito se desarrolló mediante actividades planteadas en plataforma virtual, obteniendo los siguientes resultados: el 80,5\% afirman que adquieren aprendizajes y competencias que requieren para desempeñarse en su vida profesional; no obstante, el 4,1\% no logran lo necesario. De la misma manera el 14,6\% mantiene una posición neutral en este criterio. Con los resultados verificados se afirma que se está cumpliendo con la etapa de adquisición de competencias en alto porcentaje; pero queda la reflexión ¿Qué aspectos se debe mejorar o fortalecer para que todos los docentes alcancen las competencias que demandan?

\subsection{Planificación del trabajo asistido}

El 91,8\% del alumnado afirma que la planificación y ejecución del trabajo presencial es óptima para alcanzar los resultados de aprendizaje esperados en la malla curricular, cumpliendo con el enfoque de aula invertida como complemento al proceso enseñanza-aprendizaje y fortaleciendo las competencias que el perfil docente requiere; sin embargo el 5,7\% opinan que las actividades de la jornada no cumplen con los resultados de aprendizaje; además, el $2,4 \%$ se mantiene en una posición neutral, sin sesgarse al lado positivo o negativo del criterio. Relacionando con la planificación del aprendizaje autónomo y aprendizaje experimental se deduce que el porcentaje de alumnado que incumplieron con la presentación de este componente, no está de acuerdo con las estrategias desarrolladas en la clase presencial; dado que no tienen conocimientos previos que obligatoriamente necesitan para participar y aportar perjudicando su instrucción. Con base a esto se asevera que el trabajo generado en la jornada presencial ayuda y promueve el logro de los objetivos proyectados en la etapa de la aplicación de conocimientos; generando espacios para compartir, consolidar y exponer lo aprendido.

\subsection{Orientación académica del docente en aprendizaje asistido y virtual}

Este parámetro tiene la finalidad de proporcionar datos objetivos al docente para mejorar las orientaciones académicas que realiza por medio del feedback y feedforward; el $91 \%$ del estudiantado afirman que la orientación del profesor tanto en la jornada presencial y plataforma virtual cumple con las necesidades académicas presentadas; el 4,9\% opina que el feedforward solamente se queda en feedback, faltando el complemento de retroalimentación constructiva que permita mejorar o reorientar futuras tareas; el 4,1\% se queda en la zona neutral, con lo que se deduce que están indecisos sobre el criterio. La dinámica de los resultados se repite en las variables analizadas anteriormente; lo cual es producto del incumplimiento de tareas en plataforma virtual en tiempos establecidos. Por consiguiente, se afirma que el feedforward está alcanzando su objetivo; a pesar de ello, es importante investigar la manera de mejorar este proceso.

\subsection{Estrategias motivacionales}

Como parte de las estrategias motivacionales recomendadas en el modelo AIM, a más del feedforward se realizó charlas sobre la actitud, técnicas de planificación 
tiempo- familia-estudio y ejercicios de Inteligencia Emocional.

Como complemento a las estrategias se efectuaron dinámicas grupales como: esta es mi casa, montaña rusa, colocarse por edades, exposición de metas, sueños, instrucciones para fortalecer la inteligencia emocional, entre otras; de tal forma que se pudo mantener la atención e interacción permanente, puesto que la carga horaria, espacio y tiempo son agotadoras para los estudiantes; ya que desempeñan funciones laborales durante la semana.

Frente a esto el $91,1 \%$ de estudiantes exponen que las estrategias ejecutadas permiten fortalecer la motivación, asistencia y permanencia en el estudio; el 5,7\% afirman que influye muy poco o nada; el 3,3\% se mantiene en una posición neutral. De acuerdo a los resultados analizados se aplicó una entrevista a los estudiantes que no están de acuerdo con este criterio; deduciendo que su postura es porque requieren mayor tiempo para este tipo de actividades; puesto que son padres de familia, sostén de hogar, tienen problemas causados por el estrés laboral; por lo tanto, necesitan revitalizar su parte física y emocional.

Desde esta perspectiva, resulta que las estrategias motivacionales propuestas como parte del modelo AIM, están proporcionando los resultados esperados; empero, es importante indagar nuevas estrategias para ayudar a todos los estudiantes y evitar el porcentaje negativo.

\subsection{Eficacia Modelo AIM}

El proceso desarrollado mediante el enfoque de aula invertida y motivación; tiene el 85,3\% de aceptación; el $4,1 \%$ no acepta y el $10,7 \%$ se mantiene en un estado neutral. Con base a la entrevista que se desarrolló para analizar los porcentajes negativos, el alumnado que no está de acuerdo, sugiere otra metodología que proporcione más tiempo a la parte motivacional y menos a la curricular; sin embargo, no es posible acogerla; puesto que es indispensable desarrollar competencias y resultados del plan de estudio, lo recomendable es trabajar ambos aspectos paralelamente. Finalmente se aprecia que el modelo está alcanzando los objetivos programados; considerando que un porcentaje significativo aprueba la metodología implementada, lo cual lleva a recomendar la aplicabilidad del modelo AIM de manera permanente y en otras asignaturas.

\section{Conclusiones}

La ejecución del enfoque de aula invertida puede ser articulada con estrategias motivacionales, para desarrollar competencias que respondan a las necesidades sociales y curriculares.

Las técnicas feedback, feedforward y motivación son indispensables en el proceso de enseñanza-aprendizaje a partir del enfoque de aula invertida, respondiendo a las demandas de la educación semipresencial-distancia en estudiantes adultos.
Las actividades autónomas y de praxis contribuyen a alcanzar y/o fortalecer competencias indispensables en la labor docente.

Las estrategias motivacionales promueven el aprendizaje significativo; atraen la atención y fomentan la asistencia a las jornadas presenciales, promueven la reflexión y fortalecen emociones, mejorando el estado de ánimo y consecuentemente la actitud.

El modelo AIM cumple con los requerimientos que reclama la educación semipresencial-distancia en estudiantes adultos, se fundamenta en estrategias y recursos necesarios para desenvolverse en el aspecto académico, tecnológico y motivacional; al mismo tiempo, alcanza los objetivos de aprendizaje, competencias, destrezas y motivación que necesitan los estudiantes de manera integral.

\section{Recomendaciones}

Elaborar un manual en el que se explique de manera sistemática ejemplificada el modelo AIM, de tal forma que los docentes puedan acceder a una guía precisa para su ejecución en la práctica.

Investigar otras estrategias motivacionales que cumpla con las expectativas de todos los estudiantes, entendiendo que es una población adulta que necesita fortalecer varios aspectos emocionales.

Aplicar el Modelo de Aula Invertida y Motivación (AIM) con estudiantes universitarios o adolescentes en modalidad presencial, para verificar si la propuesta tiene incidencia en otros contextos educativos.

\section{Referencias Bibliográficas}

Aguayo, M., Bravo, M., Nocetti, A., Concha, L., y Aburto, R. (2019). Perspectiva estudiantil del modelo pedagógico flipped classroom o aula invertida en el aprendizaje del inglés como lengua extranjera. Revista Educación, 43(1). doi: https://doi.org/10.15517/ revedu.v43i1.31529

Álvarez, F., Peréz, Á., , y and. (2015). MODELO PEDAGÓGICO DE LA UNIVERSIDAD NACIONAL DE EDUCACIÓN. Universidad Nacional de Educación.

Beltrán, J. (1998). PROCESOS, ESTRATEGIAS Y TÉCNICAS DE APRENDIZAJE. Madrid: Editorial Síntesis.

Bozal, M. G. (2006, jun). ESCALA MIXTA LIKERTTHURSTONE. ANDULI, Revista Andaluza de Ciencias Sociales, O(5), 81-95-95.

Canabal, C., y Margalef, L. (2017). La Retroalimentación: La Clave Para Una Evaluación Orientada Al Aprendizaje. Profesorado. Revista de Currículum y Formación de Profesorado, 21(2), 149-170.

Casas, J., Repullo, J., y Donado, J. (2003). La encuesta como técnica de investigación. Elaboración de cuestionarios y tratamiento estadístico de los datos (I). Aten Primaria, 31, 527-538.

De la Torre-Laso, J. (2019). La retroalimentación evaluativa o feedback para los trabajos en grupo como 
estrategia de acción tutorial en la universidad. $R e$ vista Educación, 43(1), 1-18. doi: https://doi.org/ 10.15517/revedu.v43i1.30062

Duarte-Herrera, M., Montalvo, D., y Valdes, D. (2019). Vista de Estrategias disposicionales y aprendizajes significativos en el aula virtual. Revista Educación, 43(2), 1-15. doi: https://doi.org/10.15517/revedu .v43i2.34038

Dutra, J., De Sousa, G., y Antonio, L. (2017). Using Technology Driven Flipped Class to Promote Active Learning in Accounting. Revista Universo Contabil; Blemenau, 13(1), 49-64. doi: http://dx.doi.org/10 $.4270 /$ ruc. 2017103

Espín, M., y Zambrano, P. (2018). La Aplicación Del Conocimiento Como Propulsor De Su Valor. Revista Científica ECOCIENCIA; Guayaquil, 5(3), 1-16.

Fuentes, L., y Pérez, L. (2019). Convivencia escolar: una mirada desde las familias. Telos, 21(1), 61-85.

Gaviria-Rodríguez, D., Arango-Arango, J., Valencia-Arias, A., y Bran-Piedrahita, L. (2019, apr). Percepción de la estrategia aula invertida en escenarios universitarios. Revista Mexinana de Investigación Educativa, 24(81), 593-614.

Goleman, D. (2007). La Inteligencia Emocional. México: ZETA.

Hernández-Silvaa, C., y Tecpan, S. (2017). Aula invertida mediada por el uso de plataformas virtuales: un estudio de caso en la formación de profesores de física. Estudios Pedagógicos, XLIII(3), 193-204.

Kriscautzky, M., y Ferreiro, E. (2014, dec). La confiabilidad de la información en Internet: criterios declarados y utilizados por jóvenes estudiantes mexicanos. Educação e Pesquisa, 40(4), 913-934. doi: 10.1590/ s1517-97022014121511

Martínez-Olvera, W., Esquivel-Gámez, I., y Martínez, J. (2014). Aula Invertida o Modelo Invertido de Aprendizaje: Origen, Sustento e Implicaciones. Los Modelos Tecno-Educativos, revolucionando el aprendizaje del siglo XXI, 1, 143-160.

Montilla, L., y Arrieta, X. (2015). Secuencia didáctica para el aprendizaje significativo del análisis volumétrico. Omnia, 21(1), 66-79.

Paredes, J., y Dias, R. (2012). La motivación del uso de las TIC en la formación de profesorado en educación ambiental. Ciência \& Educação (Bauru), 18(2), 353368.

Parrales, S., y Solórzano, J. (2014). Vista de Motivación y estrategias de aprendizaje del estudiantado de la escuela de Orientación y Educación Especial / Motivation and learning strategies of Counseling and Special Education students. Actualidades Investigativas en Educación, 14(1), 1-20.

Pinchao, L. (2016). Hacia una práctica evaluativa que favorezca el aprendizaje y mejore la enseñanza. Revista
UNIMAR, 34(1), 57-69.

Reyes, F., Vera, L., y Colina, E. (2014). Estrategias creativas para promover el aprendizaje significativo en la práctica docente simulada. Opción, 30(75), 5574.

Rojas -Lamorú, I., y Matos-Columbié, C. (2015). El desarrollo de la motivación para aprender en la Educación Superior. EduSol, 15(53), 63-69.

Salgado, J., Lería, F., Pilar, M. E., Gajardo, X., y Olivares, M. (2017). Efecto de la Motivación sobre la Profundidad en los Procesos de Estudio en Universitarios de Formación en Pedagogía. REICE. Revista Iberoamericana sobre Calidad, Eficacia y Cambio en Educación, 15.4. doi: 10.15366/reice2017.15.4.005

Schmitz, E. X. d. S., y dos Reis, S. C. (2018). Sala de aula invertida: investigação sobre o grau de familiaridade conceitual teórico-prático dos docentes da universidade. Educação Temática Digital; Campinas, 20(1), 153-175.

Sobrino, Á. (2014). Aportaciones del conectivismo como modelo pedagógico post-constructivista. Propuesta Educativa, 2(42), 39-48.

Tristán, A., y Mendoza, L. (2016). Taxonomías sobre creatividad. Revista de Psicología, 34(1), 147-183. doi: http://dx.doi.org/10.18800/psico.201601.006

UNESO. (2019). La Educación Transforma vidas.

UNICEF. (2019). Educación.

Universidad de Córdova. (2010). EL ANÁLISIS DE DATOS MEDIANTE PROCEDIMIENTOS INFORMÁTICOS Introducción al SPSS. Universidad de Córdova.

Valenzuela, J., Valenzuela, C., Silva-Peña, I., Gómez, V., y Precht, A. (2015). Motivación escolar: Claves para la formación motivacional de futuros docentes*. Estudios Pedagógicos, XLI(1), 351-361.

Zainuddin, Z., y Halili, S. H. (2016). Flipped Classroom Research and Trends from Different Fields of Study. The International Review of Research in Open and Distributed Learning, 17. doi: 10.19173/irrodl.v17i3 .2274
Recibido: 5 de septiembre de 2019

Aceptado: 12 de marzo de 2020 
